\title{
Harnessing Interference with an Out-of-Band Relay: an Approximate Capacity Result
}

\author{
Ye Tian and Aylin Yener \\ Wireless Communications and Networking Laboratory \\ Electrical Engineering Department \\ The Pennsylvania State University, University Park, PA 16802 \\ yetian@psu.edu_yener@ee.psu.edu
}

\begin{abstract}
This work studies the Gaussian interference channel (IC) with an out-of-band relay (OBR) from an informationtheoretical perspective. The relay works in bands orthogonal to the IC. The focus is on a symmetric channel model, in order to understand the fundamental impact of the OBR on the signal interaction of the IC, in the simplest possible setting. We classify the interference links as extremely strong, very strong, strong, moderate, weak, and very weak. We find that interference is useful to improve the achievable rates when it is extremely strong or very strong. Specifically, when interference is extremely strong, the channel acts as if there are two disjoint OBRs helping each source-destination pair. For strong interference, separate encoding, i.e., transmitting independent messages thorough the IC and OBR, is optimal, while for moderate interference, separate encoding is close to optimal. For weak or very weak interference, we show that the OBR can help improve the rates for common messages and further reduce the interference. It is shown that our strategy achieves the symmetric capacity to within 1.14625 bits per channel use for all channel parameters.
\end{abstract}

\section{INTRODUCTION}

Interference is a crucial factor that impacts the capacity of the wireless networks. Interference channel (IC), which consists of two source-destination pairs, is the simplest model that characterizes the effect of interference in a wireless network. To mitigate the effect of interference, recent efforts [1]-[8] considered using a relay in the interference channel, which is termed as interference relay channel (IFRC), and obtained some interesting results. References [1] proposed an interference forwarding approach and showed the benefit of decoding interference. References [3], [5], [8] proposed decode-andforward (DF) and compress-and-forward (CF) based signal relaying scheme to combat interference. References [2], [6] derived sum rate upperbounds, which complement each other, for the Gaussian IFRC. The capacity of IFRC is only known for special cases [1], [2]. For the general IFRC, the capacity is still open, since it inherits the challenges of both interference channel (IC) and relay channel (RC), with increased signal interaction.

To simplify the channel model and understand the fundamental effect of signal relaying and interference forwarding, reference [4] proposed a model where the relay operates in bands orthogonal to the IC, and the incoming links of the relay are orthogonal to its outgoing links. The relay is termed an out-of-band relay (OBR), and its operating channel, which is orthogonal to the IC, is called the out-of-band relay channel (OBRC). The sources and destinations have access to both the IC and the OBRC. This model can describe an OFDM based wireless network, where some subcarriers are dedicated to a relay, or a wireless local area network (WLAN) with Bluetooth enabled for relaying data.

Reference [4] has studied the optimality conditions of signal relaying and interference forwarding with separable or nonseparable encoding between IC and OBRC. The achievable strategies proposed therein do achieve the sum capacity for certain channel parameters, yet can also be far from outerbounds for some channel settings. It is desirable to ascertain the impact of an OBR on the signal interaction and the capacity in this model uniformly for all channel settings. This would provide us with insights on the optimal strategy to harness interference with an OBR in the wireless network, and is the main goal of our work.

For the symmetric deterministic IC with an OBR, we have recently characterized the sum capacity for all channel parameters in [7]. In this paper, we utilize the insights obtained from the deterministic model to construct achievable strategies for the Gaussian channel. We classify the interference links as extremely strong, very strong, strong, moderate, weak, and very weak. When interference is very strong or extremely strong, we find that interference is useful to improve the achievable rates. This is because the sources can transmit additional messages through the interference links, which serve as side information to help decoding of the messages transmitted through the OBRC. In particular, when interference is extremely strong, the channel acts as if there are two disjoint OBRCs helping each source-destination pair. When interference is strong, we show that separate encoding between IC and OBRC is optimal. When interference is moderate, we show that using HanKobayashi (HK) scheme for the IC and transmitting independent messages through OBRC achieves within constant gap of the outerbounds, i.e., separate encoding is near optimal. When interference is weak or very weak, using HK scheme for the IC limits the achievable rates for the common messages, since all common messages need to be decoded at interference link. We show that instead of decoding all common messages from the IC as in [9], with the OBR, part of the common messages from the interference link can be decoded from the OBRC to 


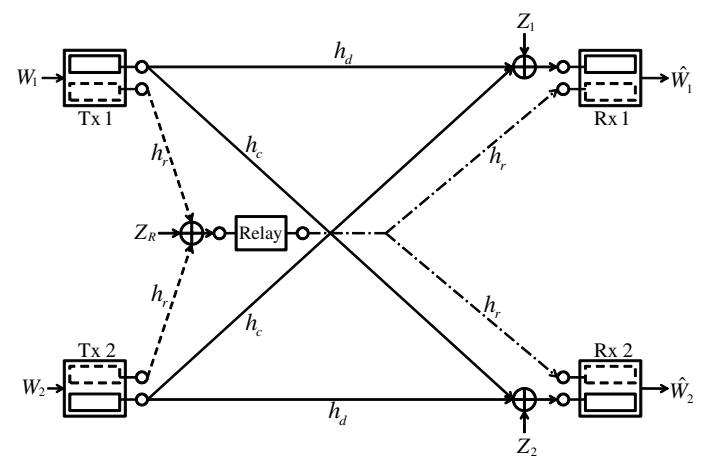

Fig. 1. The Gaussian interference channel with an out-of-band half-duplex relay.

improve the rates and further mitigate interference. By deriving new outerbounds, we show that our scheme achieves rates that are within 1.14625 bits of the symmetric capacity.

\section{Channel Model}

The Gaussian IC-OBR is shown in Figure 1, where for the interference channel, the gain of the direct link is $h_{d}$ and the gain of the interfering link is $h_{c}$. The gain of the links associated with the relay is $h_{r}$.

To communicate with its destination, source $i$ encodes a message $W_{i} \in\left\{1,2, \cdots, 2^{n R_{i}}\right\}$ into a set of codewords $\left\{X_{i}^{n}, X_{i R}^{\alpha n}\right\}$, where $X_{i}^{n}$ is the codeword for the IC while $X_{i R}^{\alpha n}$ is the codeword for the OBRC, and $\alpha$ is the duplexing factor. We assume $\alpha=0.5$ for simplicity. We assume separate power constraints on the IC and OBRC, i.e., $\frac{1}{n} \sum_{t=1}^{n} E\left[X_{i, t}^{2}\right] \leq P_{i}, \frac{1}{\alpha n} \sum_{t=1}^{\alpha n} E\left[X_{i R, t}^{2}\right] \leq P_{i R}$. The relay generates codewords based on the signals received from its incoming bands, i.e., $X_{R, \alpha n+1}^{n}=f_{R}\left(Y_{R}^{\alpha n}\right)$ with power constraints $\frac{1}{(1-\alpha) n} \sum_{t=\alpha n+1}^{n} E\left[X_{R, t}^{2}\right] \leq P_{R}$. The channel outputs for the IC are

$$
\begin{aligned}
& Y_{1, t}=h_{d} X_{1, t}+h_{c} X_{2, t}+Z_{1, t} \\
& Y_{2, t}=h_{c} X_{1, t}+h_{d} X_{2, t}+Z_{2, t}
\end{aligned}
$$

for $t=1, \cdots, n$. The channel outputs at the relay are

$$
Y_{R, t}=h_{r} X_{1 R, t}+h_{r} X_{2 R, t}+Z_{R, t}
$$

for $t=1, \cdots, \alpha n$. The channel outputs for the OBRC are

$$
\begin{aligned}
& Y_{1 R, t}=h_{r} X_{R, t}+Z_{1 R, t} \\
& Y_{2 R, t}=h_{r} X_{R, t}+Z_{2 R, t}
\end{aligned}
$$

for $t=\alpha n+1, \cdots, n$. With out loss of generality, we assume $P_{i}=P_{i R}=P_{R}=1$, and $Z_{j, t}(j=1,2,1 R, 2 R, R)$ are independent, unit variance Gaussian random variables.

\section{ApPRoXimate CAPACITy Results}

In a recent work [7], we studied the deterministic ICOBR for the symmetric case and established the sum capacity for all possible channel parameters. Specifically, the strength of the interference links are classified as extremely strong, very strong, strong, moderate, weak, and very weak. The achievable strategies for this model work as follows: For strong interference, we use separate encoding between the IC and OBRC. For moderate interference, we use Han-Kobayashi (HK) scheme and separate encoding between IC and OBRC. For the remaining cases, we send the "sum" of parts of the source messages through the OBRC. For extremely strong and very strong interference, we use the interference link of the IC to facilitate decoding of the messages transmitted through the OBRC. For weak and very weak interference, we use the OBRC in a smart fasion to reduce the interference caused by sending common information in HK scheme.

In this section, we derive outerbounds and construct achievable strategies for the Gaussian channel based on the insights obtained from the deterministic model described above.

We consider the symmetric capacity, which is defined as $C_{\text {sym }}=\sup \{R:(R, R) \in \mathcal{C}\}$, where $\mathcal{C}$ is the capacity region. For the symmetric channel, the rate points that maximize the sum rate achieve the symmetric capacity. We thus focus on the sum capacity of this channel, which, in turn, gives us results on the symmetric capacity.

Proposition 1: When the interference links are stronger than the direct links, i.e., $h_{c}^{2} \geq h_{d}^{2}$, the following expressions provide sum rate outerbounds:

$$
\begin{aligned}
& C_{\text {sum }, 1}=\log \left(1+h_{d}^{2}\right)+\frac{1}{2} \log \left(1+h_{r}^{2}\right) \\
& C_{\text {sum }, 2}=\frac{1}{2} \log \left(1+h_{c}^{2}+h_{d}^{2}\right)+\frac{1}{4} \log \left(1+h_{r}^{2}\right)
\end{aligned}
$$

When the interference links are weaker than the direct links, the following expressions provide sum rate outerbounds:

$$
\begin{gathered}
C_{\text {sum }, 3}=\log \left(1+h_{c}^{2}+\frac{h_{d}^{2}}{1+h_{c}^{2}}\right)+\frac{1}{2} \log \left(1+h_{r}^{2}\right) \\
C_{\text {sum }, 4}=\frac{1}{2} \log \left(1+h_{d}^{2}\right)+\frac{1}{2} \log \left(1+\frac{h_{d}^{2}}{1+h_{c}^{2}}\right) \\
+\frac{1}{4} \log \left(1+h_{r}^{2}\right)
\end{gathered}
$$

Sketch of the Proof: The bound (6) is due to the cut set bound. The bound (7) is obtained using the strong interference condition and symmetry of the OBRC links. The bounds (8) and (9) are obtained using genie-aided approach. The prelog factor $\frac{1}{4}$ is due to the duplexing factor. For the complete proof, see [10].

For achievability, we focus our study on the cases when $h_{d}^{2} \geq 1, h_{c}^{2} \geq 1$. We present our results for the following cases. Due to space limits, we only provide the results and describe the outline of our strategies. For detailed derivations, explanations and proofs, see [10].

A. When interference link is stronger than direct link: $h_{c}^{2} \geq h_{d}^{2}$

1) $h_{c}^{2} \leq h_{d}^{2}+h_{d}^{4}$ : For this case, we term the interference as "strong". Using separate encoding between the IC and OBRC, we can show that the following sum rate is achievable, which 
matches the outerbound $C_{\text {sum }, 2}$ :

$$
\frac{1}{2} \log \left(1+h_{d}^{2}+h_{c}^{2}\right)+\frac{1}{4} \log \left(1+h_{r}^{2}\right)
$$

The details are omitted since the scheme is a simple extension of the scheme for IC with strong interference. Note that here the relay and destinations decode both source messages transmitted through the OBRC.

2) $h_{c}^{2}>h_{d}^{2}+h_{d}^{4}$ : For this set of channel conditions, we term the interference link as "very strong" or "extremely strong", depending on the relationship between the interference links, direct links and the links in the OBRC. For this case, the sources can use the OBR in a more "smart" fashion with the help of the interference links.

Each source splits the message $W_{i}$ into $W_{i D}$ and $W_{i C}$ with rates $R_{i D}$ and $R_{i C}$, where $W_{i D}$ is to be decoded from both the direct link and the interference link, and $W_{i C}$ is to be decoded only at the interference link. The sources then encode $W_{i D}$, $W_{i C}$ into $U_{i}^{n}, V_{i}^{n}$ respectively, where $U_{i}, V_{i}$ are independent unit variance Gaussian random variables. The signals sent into the IC are

$$
X_{i}=\beta U_{i}+\sqrt{1-\beta^{2}} V_{i}
$$

To guarantee that the messages $W_{i C}$ arrive at the noise level at the direct links, we set $\beta^{2}=\frac{h_{d}^{2}-1}{h_{d}^{2}}$. Therefore, the received signal at receiver 1 from the IC is

$$
Y_{1}=\sqrt{h_{d}^{2}-1} U_{1}+\frac{h_{c}}{h_{d}} \sqrt{h_{d}^{2}-1} U_{2}+\frac{h_{c}}{h_{d}} V_{2}+Z_{1}+Z_{V_{1}}
$$

The destination 1 decodes the messages in the following order: $U_{2}\left(W_{2 D}\right) \rightarrow V_{2}\left(W_{2 C}\right) \rightarrow U_{1}\left(W_{1 D}\right)$. Note that the message $W_{1 C}$ cannot be decoded from the IC.

Since the deterministic model in [7] suggests us to send the sum of the messages through the OBRC, we use nested lattice codes. Specifically, we choose a pair of nested lattice codes $\Lambda \subset \Lambda_{c} \subset \mathbb{R}^{n}$ with nesting ratio $R_{i C}$, such that the coarse lattice $\Lambda$ is Rogers-good and Poltyrev-good [11], and the fine lattice $\Lambda_{c}$ is Poltyrev-good. Moreover, we choose the coarse lattice such that $\sigma^{2}(\Lambda)=1$. The codewords are the fine lattice points that are within the fundamental Voronoi region of the coarse lattice. Source $i$ maps the message $W_{i C}$ into a lattice point $t_{i}^{n} \in \Lambda_{c} \bigcap \mathcal{V}(\Lambda)$, and transmits $X_{i R}^{n}=\left(t_{i}^{n}+D_{i}^{n}\right)$ $\bmod \Lambda$ into the OBRC, where $D_{i}^{n} \sim \operatorname{Unif}(\mathcal{V}(\Lambda))$ is the dither. It can be shown that $X_{i R}^{n}$ satisfies the power constraint and is independent of $t_{i}^{n}[11]$.

The relay first decodes the modulo sum of the transmitted lattice points from the incoming bands, and then transmits the modulo sum of the two lattice points, which is still a lattice point, to the destinations using the outgoing bands.

At destination 1 , since it already knows $W_{2 C}$, it can now recover $W_{1 C}$ from the modulo-sum of the lattice points received from the OBRC. The decoding process of destination 2 is the same as destination 1 . We can show that when the following condition holds

$$
\left(\frac{1}{4} \log \left(\frac{1}{2}+h_{r}^{2}\right)\right)^{+} \leq \frac{1}{2} \log \left(1+\frac{h_{c}^{2}}{h_{d}^{4}+h_{d}^{2}}\right)
$$

the achieved sum rate is

$$
\log \left(1+\frac{h_{d}^{2}-1}{2}\right)+\left(\frac{1}{2} \log \left(\frac{1}{2}+h_{r}^{2}\right)\right)^{+}
$$

It is easy to verify that the gap between this rate and $C_{\text {sum }, 1}$ is $\frac{1}{2} \log 6=1.2925$ bits.

When the condition (13) holds, we term the interference link as "extremely strong". When it does not hold, we term the interference link as "very strong".

From the expression for very strong interference, we can see that some resources of the OBRC (related to $h_{r}$ ) are left unused. Based on the achievable strategy described above, the sources can encode additional messages $W_{i R}$ into $U_{i R}^{n}$, and transmit $X_{i R}^{\prime}=\gamma X_{i R}+\sqrt{1-\gamma^{2}} U_{i R}$ into the OBRC. The relay first decodes $U_{1 R}$ and $U_{2 R}$ by treating $X_{i R}$ as noise. It then subtracts $U_{i R}$ from the received signal, and decodes the modulo sum of the lattice points representing $W_{1 C}$ and $W_{2 C}$. We denote the modulo sum of these lattice points by $T_{R}$. The relay transmits the following signal to the destinations through its outgoing bands

$$
X_{R}=\gamma T_{R}+\sqrt{\frac{1-\gamma^{2}}{2}}\left(U_{1 R}+U_{2 R}\right)
$$

The destinations follow the same decoding order as the relay, i.e., they first decode $U_{1 R}, U_{2 R}$ as a MAC, and then decode $T_{R}$. We set the parameter $\gamma$ such that the rate constraints of the message $W_{i C}$ are the same for both the IC and the OBRC, i.e.,

$$
\frac{1}{4} \log \left(\frac{1}{2}+h_{r}^{2} \gamma^{2}\right)=\frac{1}{2} \log \left(1+\frac{h_{c}^{2}}{h_{d}^{4}+h_{d}^{2}}\right)
$$

We can show that the achieved sum rate is

$\log \left(1+\frac{h_{d}^{2}-1}{2}\right)+\log \left(1+\frac{h_{c}^{2}}{h_{d}^{4}+h_{d}^{2}}\right)+\frac{1}{4} \log \left(\frac{1+h_{r}^{2}}{1+h_{r}^{2} \gamma^{2}}\right)$

It can be shown that the gap with the bound $C_{s u m, 2}$ is thus at most 1.25 bits.

B. When interference link is weaker than direct link: $h_{c}^{2}<h_{d}^{2}$

1) $h_{d}^{2}\left(h_{d}^{2}+h_{c}^{2}\right) \leq h_{c}^{4}\left(h_{c}^{2}+1\right)$ : For this case, we term the interference link as "moderate". Based on a simple HanKobayashi (HK) strategy for the IC used in reference [9] and separate encoding between the IC and the OBRC, we can show that the following sum rate is achievable

$\frac{1}{2} \log \left(1+h_{d}^{2}+h_{c}^{2}\right)+\frac{1}{2} \log \left(2+\frac{h_{d}^{2}}{h_{c}^{2}}\right)-1+\frac{1}{4} \log \left(1+h_{r}^{2}\right)$

It is easy to verify that the gap between this rate and the outerbound $C_{\text {sum, } 4}$ is 1 bit. Note that here the relay and the destinations decode both source messages transmitted through the OBRC.

2) $h_{d}^{2}\left(h_{d}^{2}+h_{c}^{2}\right)>h_{c}^{4}\left(h_{c}^{2}+1\right) \geq\left(h_{d}^{2}+h_{c}^{2}\right)\left(h_{c}^{2}+1\right)$ : For this case, we term the interference link as "weak". In light of the results from [7], we first split the message $W_{i}$ into $W_{i c}$ and $W_{i p}$. We further split $W_{i c}$ into $W_{i c a}$ and $W_{i c b}$, and encode $W_{i c a}, W_{i c b}$ and $W_{i p}$ into $U_{i a}^{n}, U_{i b}^{n}, V_{i}^{n}$ respectively, 
where $U_{i a}, U_{i b}, V_{i} \sim \mathcal{N}(0,1)$. The sources thus transmit the following signal through the IC:

$$
X_{i}=\beta\left(\theta U_{i a}+\sqrt{1-\theta^{2}} U_{i b}\right)+\sqrt{1-\beta^{2}} V_{i}
$$

Now a critical step is how to design the power allocation parameter $\theta$. From the insights obtained from the deterministic model [7], we choose $\theta$ such that $U_{2 b}\left(U_{1 b}\right)$ and $V_{1}\left(V_{2}\right)$ are aligned at the same level at destination 1 (2). We have

$$
1-\theta^{2}=\frac{h_{d}^{2}}{h_{c}^{2}\left(h_{c}^{2}-1\right)}
$$

Note that we need $h_{c}^{4} \geq h_{d}^{2}+h_{c}^{2}$ since $\theta \in[0,1]$. We can then rewrite $Y_{1}$ as

$$
\begin{aligned}
Y_{1} & =\frac{h_{d}}{h_{c}^{2}} \sqrt{h_{c}^{4}-h_{c}^{2}-h_{d}^{2}} U_{1 a}+\frac{h_{d}^{2}}{h_{c}^{2}} U_{1 b} \\
& +\frac{\sqrt{h_{c}^{4}-h_{c}^{2}-h_{d}^{2}}}{h_{c}} U_{2 a}+\frac{h_{d}}{h_{c}}\left(V_{1}+U_{2 b}\right)+Z_{1}+V_{2}
\end{aligned}
$$

The signal $Y_{2}$ is of similar format.

At the same time, the sources also utilize the OBRC to send the messages $W_{i c b}$ using lattice codes following the construction in section III-A. The relay decodes the modulo sum of these two messages, and forwards it to the destinations.

The decoder at destination 1 decodes the signal transmitted through the IC in the following order: $U_{1 a}^{n} \rightarrow U_{1 b}^{n} \rightarrow U_{2 a}^{n}$. The decoder also decodes the signal transmitted through the OBRC to obtain the modulo sum of the messages $W_{1 c b}$ and $W_{2 c b}$. Since decoder 1 knows $W_{1 c b}$ from decoding $U_{1 b}$, it can recover $W_{2 c b}$. The interference signal $U_{2 b}$ then can be subtracted from $Y_{1}$, and $V_{1}$ can be decoded. The decoder at destination 2 uses the same decoding method. We can show that when the following condition holds

$$
\frac{1}{2} \log \left(1+\frac{h_{d}^{4}}{h_{c}^{6}+h_{c}^{4}+h_{d}^{2} h_{c}^{2}}\right) \geq\left(\frac{1}{4} \log \left(\frac{1}{2}+h_{r}^{2}\right)\right)^{+}
$$

the achievable sum rate is

$$
\begin{aligned}
& \log \left(1+\frac{h_{d}^{2}}{2 h_{c}^{2}}\right)+\log \left(1+\frac{h_{c}^{4}-h_{c}^{2}-h_{d}^{2}}{2 h_{d}^{2}+2 h_{c}^{2}}\right) \\
& \quad+\left(\frac{1}{2} \log \left(\frac{1}{2}+h_{r}^{2}\right)\right)^{+}
\end{aligned}
$$

It can be verified that the gap between this rate and the outerbound $C_{\text {sum }, 3}$ is at most $\log 2 \sqrt{6}=2.2925$ bits.

From the condition (22), we can see that the resources in the OBRC (related to $h_{r}$ ) are limited, and they are all used to help the destinations decode part of the common message. No resource can be utilized to send new information. When (22) does not hold, there are additional resources available and the achievable scheme can be further improved by sending new messages $W_{i R}$ in addition to $W_{i c b}$ through the OBRC following similar steps as in the case of very strong interference.
Using this approach, the achievable sum rate is

$$
\begin{aligned}
& \log \left(1+\frac{h_{d}^{2}}{2 h_{c}^{2}}\right)+\log \left(1+\frac{h_{c}^{4}-h_{c}^{2}-h_{d}^{2}}{2 h_{d}^{2}+2 h_{c}^{2}}\right) \\
& +\log \left(1+\frac{h_{d}^{4}}{h_{c}^{6}+h_{c}^{4}+h_{d}^{2} h_{c}^{2}}\right)+\frac{1}{4} \log \left(\frac{1+h_{r}^{2}}{1+h_{r}^{2} \gamma^{2}}\right)
\end{aligned}
$$

where we choose the parameter $\gamma$ such that the rate constraints for $W_{i c b}$ are the same at the IC and the OBRC, which requires the following condition

$$
\frac{1}{2} \log \left(1+\frac{h_{d}^{4}}{h_{c}^{6}+h_{c}^{4}+h_{d}^{2} h_{c}^{2}}\right)=\frac{1}{4} \log \left(\frac{1}{2}+h_{r}^{2} \gamma^{2}\right)
$$

It can be verified that the gap between this rate and the outerbound $C_{\text {sum }, 4}$ is at most 2.25 bits.

3) $h_{c}^{4}<h_{d}^{2}+h_{c}^{2}$ : For this case, we term the interference link as "very weak". The rate splitting strategy in section III-B2 does not work for this range of channel parameters. The idea of the achievable strategy for this case is similar to the one we used for weak interference. We thus only give an outline of the achievable scheme and focus on the difference.

The sources split the message into common and private parts and transmit them into the IC such that the signals carrying the private message arrive at noise level at the interference links. The common messages are also transmitted through the OBRC using lattice code. The destinations decode the intended source common message from the IC, and decodes the modulo-sum of two common messages from the OBRC. The interference common message can be recovered. The private message is then decoded by treating the rest interference signal as noise. We can show that the achievable sum rate is

$$
\log \left(1+\frac{h_{d}^{2}}{2 h_{c}^{2}}\right)+\left(\frac{1}{2} \log \left(\frac{1}{2}+h_{r}^{2}\right)\right)^{+}
$$

when the following condition holds

$$
\left(\frac{1}{4} \log \left(\frac{1}{2}+h_{r}^{2}\right)\right)^{+} \leq \frac{1}{2} \log \left(1+\frac{h_{d}^{2}\left(h_{c}^{2}-1\right)}{h_{c}^{4}+h_{c}^{2}+h_{d}^{2}}\right)
$$

It can be verified that the gap between this rate and the outerbound $C_{\text {sum }, 3}$ is at most 2.2925 bits.

When the condition (27) does not hold, the achievable sum rate can be improved by transmitting additional message in addition to the common messages through the OBRC following similar steps as in the case of very strong interference.

The improved achievable sum rate is

$$
\begin{aligned}
& \log \left(1+\frac{h_{d}^{2}}{2 h_{c}^{2}}\right)+\log \left(1+\frac{h_{d}^{2}\left(h_{c}^{2}-1\right)}{h_{c}^{4}+h_{c}^{2}+h_{d}^{2}}\right) \\
& +\frac{1}{4} \log \left(\frac{1+h_{r}^{2}}{1+h_{r}^{2} \gamma^{2}}\right)
\end{aligned}
$$

where the parameter $\gamma$ guarantees that the rate constraints for the common messages are the same at the IC and the OBRC.

We can show that that the gap between this sum rate and the outerbound $C_{\text {sum }, 4}$ is at most 1.75 bits. 


\section{Constant gap result for symmetric capacity}

The sum capacity is simply twice the symmetric capacity. Hence our achievable strategy achieves within 1.14625 bits of the symmetric capacity for $h_{c} \geq 1$ and $h_{d} \geq 1$. It is easy to verify that when $h_{c}<1$ or $h_{d}<1$, the result still holds. Therefore we conclude with the following theorem:

Theorem 1: The symmetric capacity $C_{\text {sym }}$ of the symmetric Gaussian IC-OBR for duplexing factor 0.5 is within 1.14625 bits of $\bar{C}_{\text {sym }}$, or

$$
\bar{C}_{\text {sym }}-1.14625 \leq C_{\text {sym }} \leq \bar{C}_{\text {sym }}
$$

where

$$
\begin{array}{ll}
\bar{C}_{\text {sym }}=\frac{1}{2} \min \left\{C_{\text {sum }, 1}, C_{\text {sum }, 2}\right\}, & h_{c}^{2} \geq h_{d}^{2} \\
\bar{C}_{\text {sym }}=\frac{1}{2} \min \left\{C_{\text {sum }, 3}, C_{\text {sum }, 4}\right\}, & h_{c}^{2}<h_{d}^{2}
\end{array}
$$

\section{Insights from the achievable strategy}

The achievable strategies and constant gap results provide us insights on how the OBR changes the signal interaction of the IC. For extremely strong and very strong interference, the interference links support much larger rates than the direct links. For the IC without OBR, the excessive rates of the interference links can only help with interference cancelation to achieve the maximum rates supported by the direct links, but they cannot be used to further improve the achievable rates. With the OBR, however, the interference links can be used to convey some side information to the destinations. This side information can facilitate the transmission through the OBRC. In particular, we observe that when the interference is extremely strong, the channel acts as if there are two disjoint OBRCs assisting each source-destination pair. This can be seen from the sum rate bound (6). Under the condition in (13), the users can achieve within 1.2925 bits of this rate. The term $\frac{1}{2} \log \left(1+h_{r}^{2}\right)$ acts as if there are two independent OBRC connecting each source-destination pair.

When interference link is weaker than the direct link, a simplified version of the HK scheme achieves within 1 bit of the capacity region for the IC without OBR [9]. The HK strategy splits the message into common and private parts, where the common message causes interference at the nonintended receivers. We call the common message from the intended source as the common information message, while the common message from the non-intended source as the common interference message, from the receiver's perspective. Without the OBR, the decoder needs to decode both the common interference and information messages from the IC to reduce the effect of the interference. For weak and very weak interference, when applying this scheme to the IC with an OBR, it has unbounded gap with the outerbounds, since the interference link is bottleneck for the achievable rates of common messages. In fact, from the strategies described in section III-B2 and III-B3, we can see that the OBR changes how the users should treat the common interference messages. When interference is very weak, decoding the common inter- ference messages from the interference link of IC imposes a severe rate constraint on the achievable rate. With the OBR, the decoders can decode the common information message from the direct link of the IC first, and then recover the common interference message from the OBRC to improve the rates. When the interference is stronger than very weak but is still weak, the interference link can support some common interference messages, and thus it is beneficial to further split the common message into two parts. Both parts of the common information message, and one part of the common interference message can be decoded from the IC. With these messages, the other part of the common interference message can be recovered from the OBRC to achieve higher rates.

\section{CONCLUSION}

In this paper, we have studied the Gaussian IC-OBR and established the symmetric capacity to within 1.15 bits for all channel parameters. We have classified the interference links as extremely strong, very strong, strong, moderate, weak, and very weak, according to the relative strength between the interference links, direct links, and links in the OBRC. By deriving outerbounds and constructing achievable strategies, we have shown that separate encoding is optimal for strong interference and near optimal for moderate interference. We have also shown that for very strong and extremely strong interference, the interference links are useful to improve achievable rates. For weak and very weak interference, we have shown that the OBRC can improve the rates for common messages and further mitigate the interference.

\section{REFERENCES}

[1] I. Maric, R. Dabora, and A. Goldsmith, "On the capacity of the interference channel with a relay," in Proceedings of IEEE International Symposium on Information Theory, July 2008.

[2] _ , "An outer bound for the Gaussian interfrence channel with a relay," in Proceedings of IEEE Information Theory Workshop, October 2009.

[3] O. Sahin and E. Erkip, "Achievable rates for the Gaussian interference relay channel," in Proceedings of 2007 GLOBECOM Communication Theory Symposium, November 2007.

[4] O. Sahin, O. Simeone, and E. Erkip, "Interference channel with a half-duplex out-of-band relay," in Proceedings of IEEE International Symposium on Information Theory, June 2010.

[5] P. Razaghi and W. Yu, "Universal relaying for the interference channel," in Information Theory and Applications Workshop (ITA), Feburary 2010.

[6] Y. Tian and A. Yener, "The Gaussian interference relay channel with a potent relay," in Proceedings of the IEEE Global Telecommunications Conference, December 2009.

[7] — "Sum capacity of the deterministic interference channel with an out-of-band half-duplex relay," in Proceedings of Forty-eighth Annual Allerton Conference On Communication, Control, and Computing, October 2010.

[8] — " "The Gaussian interference relay channel: Improved achievable rates and sum rate upperbounds using a potent relay," to appear in IEEE Transactions on Information Theory, Special Issue on Interference Networks, available at arXiv:1102.0043v1.

[9] R. H. Etkin, D. N. C. Tse, and H. Wang, "Gaussian interference channel capacity to within one bit," IEEE Transactions on Information Theory, vol. 54, no. 12, pp. 5534-5562, December 2008.

[10] Y. Tian and A. Yener, "Symmetric capacity of the Gaussian interference channel with an out-of-band relay to within 1.15 bits," submitted to IEEE Transactions on Information Theory, available at arXiv:1010.6290v1.

[11] U. Erez and R. Zamir, "Achieving $1 / 2 \log (1+S N R)$ on the AWGN channel with lattice encoding and decoding," IEEE Transactions on Information Theory, vol. 50, no. 10, pp. 2293-2314, October 2004. 